\title{
Fatty acid composition in relation to the metabolic syndrome and associated cardiovascular risk factors
}

\author{
Rachel M. Fisher and Per Sjögren \\ Atherosclerosis Research Unit, King Gustaf V Research Institute, Karolinska Institutet, Stockholm, Sweden
}

Abstract

Fatty acid metabolism is important in relation to the development of insulin resistance and the metabolic syndrome. Elevated total non-esterified fatty acid concentrations predispose to an atherogenic lipoprotein profile, but the individual properties of specific fatty acids are also of importance for the development of the metabolic syndrome. The objectives of this study were to investigate the relationships between fatty acid composition and selected cardiovascular risk factors associated with the metabolic syndrome [small, dense low-density lipoprotein (LDL) particles and oxidative stress], and to investigate estimates of adipose tissue $\Delta^{9}$-desaturase activity. A cohort of approximately 300 healthy Swedish men (aged 62-64 years), extensively characterized in relation to components of the metabolic syndrome, was investigated cross-sectionally. Increases in fatty acids typical of milk products were associated with reduced numbers of potentially atherogenic small, dense LDL particles, and n-3 fatty acids were independent (negative) predictors of oxidative stress. $\Delta^{9}$-Desaturase activity in human adipose tissue needs more detailed investigation in relation to the metabolic syndrome. In conclusion, the quality of dietary fat is of importance in relation to the development of cardiovascular risk factors associated with the metabolic syndrome.

Keywords: insulin resistance; LDL particle size; oxidative stress; stearoyl CoA desaturase

Abbreviations: ACC: acetyl-coenzyme A carboxylase; AMP: adenosine monophosphate; AMPK: AMPactivated protein kinase; ASO: antisense oligonucleotide; CETP: cholesteryl ester transfer protein; CoA: coenzyme A; CPT-1: carnitine palmitoyltransferase-1; HDL: high-density lipoprotein; 8-iso-PGF2 $\alpha$ : 8-isoprostaglandin F2 $\alpha$; LDL: low-density lipoprotein; NEFA: non-esterified fatty acid; ox LDL: oxidized low-density lipoprotein; SCD: stearoyl-coenzyme A desaturase; TG: triacylglycerol; VLDL: very low-density lipoprotein.

\section{Introduction}

A current major health problem in the Western world is the dramatic increase in the occurrence of type 2 diabetes and the associated increased risk of developing cardiovascular disease. Insulin resistance and the metabolic syndrome both greatly predispose to the development of type 2 diabetes and there is therefore great interest in identifying the factors leading to the onset of these conditions. Important components of the metabolic syndrome include insulin resistance itself, a classic dyslipidaemia [a so-called atherogenic lipoprotein profile, consisting of high triacylglycerol (TG), low high-density lipoprotein (HDL)-cholesterol concentrations and a preponderance of small, dense lowdensity lipoprotein (LDL) particles], and obesity, in particular abdominal obesity.
Lipid abnormalities associated with insulin resistance have more recently been realized to include defects in fatty acid metabolism, and elevated circulating non-esterified fatty acid (NEFA) concentrations may be a risk factor for cardiovascular disease (1). Since NEFAs can effectively compete with glucose for substrate oxidation in muscle, elevated NEFA concentrations may inhibit wholebody glucose disposal and hence induce insulin resistance. Furthermore, elevated NEFA concentrations could contribute to the development of an atherogenic lipoprotein profile. An increased flux of NEFAs to the liver may stimulate hepatic TG synthesis and enhance very low-density lipoprotein (VLDL) production. The subsequent high circulating TG concentrations drive excessive cholesteryl ester transfer protein (CETP)-mediated 
transfer of TG out of VLDL (and chylomicron) particles into LDL and HDL particles in exchange for cholesteryl ester. These TG-enriched LDL and HDL particles are favourable substrates for hepatic lipase, which hydrolyses the TG core, leaving small, dense, TG-depleted LDL and HDL particles. Therefore, high NEFA concentrations may lead to high TG and low HDL-cholesterol concentrations, and a preponderance of small, dense LDL particles. There is a well-established link between small, dense LDL and the progression of atherosclerosis and the development of cardiovascular disease (2), which may be explained by the increased susceptibility of these LDL particles to oxidation. While the uptake of native LDL by macrophages does not result in foam cell formation, the uptake of oxidized lowdensity lipoprotein (oxLDL) by scavenger receptors results in unregulated lipid accumulation. Staining for oxLDL has been demonstrated in both animal and human atherosclerotic lesions, and circulating concentrations of oxLDL were significantly higher in patients with cardiovascular disease than in controls, and oxLDL concentrations correlated with the severity of clinical presentation $(3,4)$. Indeed, a state of increased oxidative stress has been proposed as the pathogenic mechanism linking insulin resistance to the development of diabetes and cardiovascular disease (5), but how fatty acid composition and metabolism relates to oxidative stress in the metabolic syndrome is poorly understood.

In addition to the potential consequences of high circulating NEFA concentrations described above, the significance of the metabolic properties of different fatty acids needs to be considered. The fatty acid composition of dietary fat is believed to be an important contributor to the development of the metabolic syndrome. For example, insulin sensitivity was impaired in subjects in whom dietary monounsaturated fatty acids were replaced with saturated fatty acids (6). Furthermore, characteristic fatty acid composition patterns of serum phospholipids and cholesteryl esters are associated with insulin-resistant states. These patterns include increased proportions of 16:0 (palmitic acid), 16:1 (palmitoleic acid) and 20:3 n-6 (dihomo- $\gamma$-linolenic acid), and a decreased proportion of 18:2 n-6 (linoleic acid) (7). To synthesize longer, more unsaturated fatty acids a combination of desaturation and chain elongation is required, but relatively little is known about the regulation of these processes. However, fatty acid patterns indicate that high activities of $\Delta^{9}$ - and $\Delta^{6}$-desaturases and a low activity of $\Delta^{5}$-desaturase are associated with insulin-resistant states (7).

Therefore, fatty acid metabolism is important at a number of different levels in relation to the development of insulin resistance and the metabolic syndrome. Elevated total NEFA concentrations may predispose to an atherogenic lipoprotein profile, but the individual properties of specific fatty acids may also have an impact on the development of the metabolic syndrome (Fig. 1). A cohort of approximately 300 healthy Swedish men (aged 62-64 years), in whom all components of the metabolic syndrome have been characterized in detail (8), was used to investigate relationships between fatty acid composition and selected cardiovascular risk factors associated with the metabolic syndrome (small, dense LDL particles and oxidative stress). Estimates of adipose tissue $\Delta^{9}$-desaturase activity will also be investigated.

\section{Small, dense low-density lipoprotein}

A predominance of small, dense LDL particles is a central component of the atherogenic lipoprotein profile typical of the metabolic syndrome. Therefore, fatty acid composition profiles of the diet, serum and adipose tissue were investigated in relation to the occurrence of small, dense LDL particles in the cohort of healthy 63-year-old men (9). LDL particle size distribution was determined by polyacrylamide gradient gel electrophoresis, staining and subsequent scanning giving peak particle size of LDL and relative distribution of LDL in predefined subfractions with cut-offs: LDLI $(27.0-25.0 \mathrm{~nm})$, LDL-II $(25.0-23.5 \mathrm{~nm})$, LDL-III $(23.5-22.5 \mathrm{~nm})$ and LDL-IV $(22.5-21.0 \mathrm{~nm})$. Both

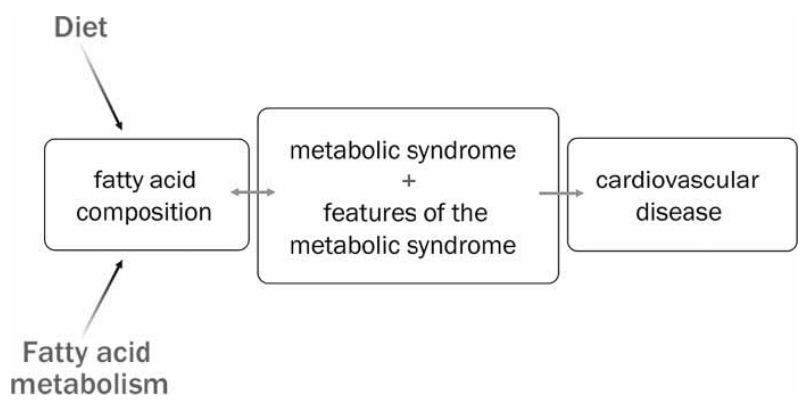

Fig. 1. Fatty acid composition is believed to contribute to the development of the metabolic syndrome and its associated features and subsequent cardiovascular disease. The spectrum of fatty acids in the body can be influenced by both the quality of dietary fat and the metabolism of fatty acids. 
subfractions III and IV contain the small, dense LDL particles, but the principal small, dense LDL subfraction is LDL-III, which comprised $18.5 \pm$ $8.9 \%$ of total LDL particles, while LDL-IV contained just $4.2 \pm 1.8 \%$. Furthermore, the LDL-III subfraction has previously been shown to be strongly related to atherosclerosis (10). Therefore, the men were divided into tertiles of percentage distribution of LDL-III, with individuals in the lowest tertile being considered to have a less atherogenic LDL profile with the least small, dense LDL particles.

The amount of LDL-III (\%) was inversely related to the reported consumption of fat from dairy

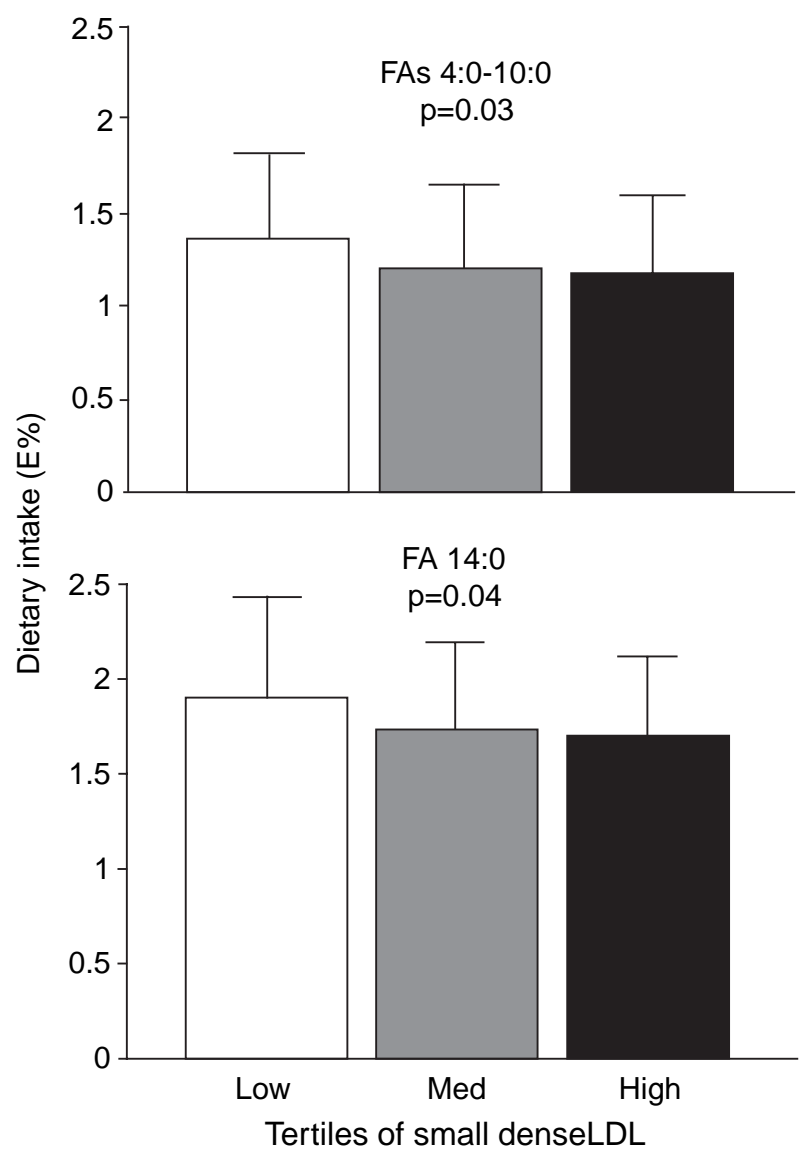

Fig. 2. High consumption of fat from dairy products is associated with a decrease in small, dense low-density lipoprotein (LDL). Healthy, 63-year-old men were divided into tertiles [Low, Med (medium) and High] of small, dense LDL [LDL-III (\%)]. Dietary consumption was estimated from 7 day dietary records and data are presented from men $(n=207)$ identified as non-underreporters of their energy intake. The estimated consumption, in energy $\%(E \%)$ of fatty acids (FAs) of chain length 4:0-10:0 (upper panel) and 14:0 (lower panel), which are found abundantly in dairy products, is shown. Men in the highest tertile of small, dense LDL-III are considered to have a more atherogenic LDL profile. The $p$-values are for ANOVA across all groups. All data are taken from Sjogren et al. (9). products and specific fatty acids (4:0-10:0 and 14:0) found abundantly in dairy products (Fig. 2) (9). In other words, men in the lowest tertile of LDL-III had the highest consumption of milk fat. The tertiles of LDL-III (\%) did not differ with respect to reported consumption of monounsaturated, polyunsaturated or saturated fatty acids, but consumption of saturated fatty acids was significantly higher in the low compared with the high LDL-III tertile group. Furthermore, the same pattern was seen for the relative amounts of myristic (14:0), pentadecanoic (15:0) and heptadecanoic (17:0) acids in serum phospholipids and serum NEFAs. In adipose tissue the same patterns were observed, but the relationships were not statistically significant. Pentadecanoic and heptadecanoic acids are fatty acids that are almost exclusively found in milk products. Therefore, differences in LDL particle size distribution were related to fatty acids specific for dairy products. Indeed, there was an apparently beneficial effect of milk products, with an increased consumption associated with reduced numbers of the potentially atherogenic small, dense LDL particles. These observations are in agreement with a number of other studies that have reported an inverse relationship between the consumption of milk products and some risk factors for cardiovascular disease, as reviewed recently (11). Furthermore, several recent studies have found a similar inverse relationship between dairy intake and development of the metabolic syndrome $(12,13)$ and type 2 diabetes (14).

Other studies, however, are harder to reconcile with these observations. For example, a diet with a high proportion of saturated fatty acids (which include the typical milk fatty acids mentioned above) was detrimental for insulin sensitivity (6) and did not appear to have any beneficial effect on LDL size (15). However, while the present study determined the distribution of LDL particles, Rivellese et al. (15) quantified only the diameter of the major LDL peak and therefore may not have been able to detect subtle changes in LDL particle size distribution. In addition, the decrease in insulin sensitivity associated with the high saturated fatty acid diet (6) was restricted to individuals with a total fat intake in the lowest $50 \%$, but whether there was any change in LDL peak size in just this subgroup was not shown (15). The findings that diets high in saturated fatty acids raise total cholesterol and LDL-cholesterol concentrations 
(16) and impair insulin sensitivity (6) may be considered contradictory to the reports (described above) of an inverse relationship between consumption of milk products and risk factors for cardiovascular disease. Furthermore, subjects who make changes in lifestyle factors, such as reducing saturated fat intake, improve their insulin sensitivity (17). One explanation for this apparent discrepancy is that changes in lifestyle can have an impact on a wide range of parameters, such as reducing body weight (17), which could, in turn, mediate improvements in insulin sensitivity. Another possible explanation is that certain saturated fatty acids, not those typical of milk products, mediate the unfavourable effects of diets high in saturated fatty acids and mask any beneficial effects of milk-derived saturated fatty acids. Indeed, whether favourable effects can be ascribed to the individual fatty acids in milk products, or whether they can be explained by other bioactive components of dairy products (e.g. calcium), or even by lifestyle factors (such as level of physical activity) related to consumption of milk products remains to be determined. Well-controlled intervention studies are required to address these issues.

\section{Oxidative stress}

To extend upon investigations of cardiovascular risk factors associated with the metabolic syndrome, the authors determined circulating concentrations of oxLDL and urinary concentrations of 8-iso-prostaglandin $\mathrm{F}_{2 \alpha}\left(8\right.$-iso- $\left.\mathrm{PGF}_{2 \alpha}\right)$ in the cohort of healthy 63 -year-old men (18). In addition to the wellrecognized cardiovascular risk factors associated with the metabolic syndrome (such as the atherogenic lipoprotein profile discussed above), "newer" risk factors, such as oxidative stress, have been proposed (5). Oxidative modification of LDL particles to produce oxLDL is believed to be an important step in the atherosclerotic process. To estimate levels of oxidative stress, quantification of $\mathrm{F}_{2}$-isoprostanes has become an accepted tool, possibly the most reliable biomarker of oxidative stress in vivo (19). The $\mathrm{F}_{2}$-isoprostanes are stable products formed from the free radical-induced peroxidation of phospholipid-bound arachidonic acid, with 8-iso-PGF ${ }_{2 \alpha}$ being the major $\mathrm{F}_{2}$-isoprostane formed during lipid peroxidation. In contrast to some other studies $(20,21)$, this group found no increases in either circulating oxLDL or urinary 8 -iso-PGF ${ }_{2 \alpha}$ concentrations in men classed (using the National Cholesterol Education Program definition) as having the metabolic syndrome (18). Furthermore, oxLDL and 8-iso- $\mathrm{PGF}_{2 \alpha}$ were not related to one another, suggesting that they represent different aspects of oxidative modification. No classes of fat or individual fatty acids entered the multivariate analysis for oxLDL, with the strongest predictors being LDL-cholesterol concentrations and LDL peak particle size. However, multivariate analysis for 8 -iso- $\mathrm{PGF}_{2 \alpha}$ identified the relative content of $n-3$ fatty acids in serum phospholipids and dietary folate as independent negative predictors. The inverse relationship between 8 -iso- $\mathrm{PGF}_{2 \alpha}$ concentrations and n-3 fatty acids in serum phospholipids is in agreement with supplementation studies with n-3 fatty acids $(22,23)$ and may be explained by the anti-inflammatory properties ascribed to the n-3 fatty acids (24) and subsequent decreased leukocyte free radical formation. However, the present study found no relationship between 8 -iso-PGF ${ }_{2 \alpha}$ concentrations and reported consumption of $\mathrm{n}-3$ fatty acids. Therefore, these data suggest that specific fatty acids can influence pathways leading to lipid peroxidation, which may in turn be of relevance for subsequent cardiovascular disease.

\section{Stearoyl coenzyme A desaturase}

The fatty acid composition of serum and adipose tissue reflects the dietary composition (albeit with different strengths for different fatty acids) during the preceding weeks and months to years, respectively (7). However, the fatty acid pattern is also dependent on the endogenous synthesis and metabolism of fatty acids. Desaturases are involved in endogenous fatty acid synthesis and one such enzyme has been investigated in some detail. Stearoyl-coenzyme A desaturase (SCD) catalyses the synthesis of monounsaturated fatty acids from saturated fatty acids, introducing the cis double bond in the $\Delta 9$ position of fatty acyl-coenzyme $\mathrm{A}$ (CoA) substrates. Its preferred substrates are palmitoyl-CoA (16:0) and stearoyl-CoA (18:0), which are converted to palmitoleoyl-CoA (16:1) and oleoyl-CoA (18:1), respectively. The principal tissues sites of SCD activity are liver and adipose tissue (25). SCD knockout mice have lower TG concentrations, reduced body adiposity and increased insulin sensitivity, and are resistant to diet-induced weight gain (26). These observations have been explained by increased phosphorylation/ 
activation of adenosine monophosphate (AMP)activated protein kinase (AMPK) leading to increased phosphorylation/deactivation of acetylcoenzyme A carboxylase (ACC), resulting in reduced concentrations of malonyl-CoA and hence increased transport of fatty acids into mitochondria via activation of carnitine palmitoyltransferase1 (CPT-1) and an increase in hepatic fatty acid $\beta$-oxidation in mice deficient in SCD (27). Furthermore, leptin has been shown to repress hepatic SCD activity (28), an effect that is independent of insulin (29). This down-regulation of SCD is believed to represent an important component of leptin's metabolic actions. More recently, SCD expression has been manipulated in mouse models through the use of in vivo antisense oligonucleotides (ASOs). ASOmediated inhibition (10 weeks) of SCD expression gave similar results to those in SCD knockout mice, with a resistance to high-fat diet-induced obesity and hepatic steatosis (30). When a much shorter term ASO-mediated inhibition of SCD expression was used (5 days), hepatic insulin resistance associated with high-fat feeding was completely reversed and this effect occurred before any changes in body weight (31). These data indicate that hepatic SCD activity is involved in the development of dietinduced hepatic insulin resistance.

$\operatorname{SCD}\left(\Delta^{9}\right.$-desaturase) activity can be estimated in humans by calculating the ratios of the relative abundance of the product/substrate fatty acids, namely $16: 1 / 16: 0$ or $18: 1 / 18: 0$, to generate a "desaturation index". This desaturation index calculated for fatty acids in serum is believed to be principally a reflection of hepatic SCD activity and has been shown to be negatively associated with insulin sensitivity (32) and to explain $44 \%$ of the variance in TG concentrations (33). Furthermore, the $\Delta^{9}$ desaturation index in serum cholesteryl esters was shown to be a predictor of obesity (34) and future development of the metabolic syndrome (35). Thus, these human data indicate that increased hepatic SCD activity is unfavourable, which is in agreement with the animal studies described above. However, whether the same applies to the activity of SCD in adipose tissue is less clear. Insulin sensitization of patients with type 2 diabetes by treatment with a thiazolidinedione increased both SCD mRNA expression and $\Delta^{9}$ desaturation index in subcutaneous adipose tissue (36). The improvement of insulin sensitivity brought about by thiazolidinedione treatment has been suggested to be mediated, at least in part, by promoting the deposition of TG in adipose tissue as opposed to in other insulin-sensitive tissues (such as liver or muscle) (37). Therefore, mechanisms promoting TG storage in adipose tissue, such as local increases in SCD activity, may be beneficial in this context. Whether adipose tissue SCD activity in healthy individuals is positively or negatively related to the metabolic syndrome remains to be established. Therefore, SCD in adipose tissue will be investigated in the study cohort of healthy men. Relationships between the $\Delta^{9}$ desaturation indices in adipose tissue and serum, and the metabolic syndrome and its associated cardiovascular risk factors will be investigated. This will provide novel data on SCD in human adipose tissue, which will be particularly interesting in light of the suggestion of SCD as a potential target for treatment of obesity and the metabolic syndrome (38).

\section{Conclusions}

The studies summarized here demonstrate that the quality of dietary fat is of importance in relation to the development of cardiovascular risk factors associated with the metabolic syndrome. Improved understanding of the impact of the fatty acid composition of the diet on the metabolic syndrome and related cardiovascular risk factors is of importance from a public health perspective. Furthermore, a greater knowledge of the pathways governing endogenous fatty acid synthesis and metabolism is required to understand more fully the roles played by individual fatty acids.

\section{Acknowledgements}

Work performed by the authors is supported by grants from the Swedish Research Council (project 15352), the Swedish Diabetes Association and the Swedish Nutrition Foundation.

\section{References}

1. Carlsson M, Wessman Y, Almgren P, Groop L. High levels of nonesterified fatty acids are associated with increased familial risk of cardiovascular disease. Arterioscler Thromb Vasc Biol 2000; 20: 1588-94.

2. Superko RH. Lipoprotein subclasses and atherosclerosis. Front Biosci 2001; 6: D355-65.

3. Tsimikas S, Witztum JL. Measuring circulating oxidized low-density lipoprotein to evaluate coronary risk. Circulation 2001; 103: 1930-2.

4. Ehara S, Ueda M, Naruko T, Haze K, Itoh A, Otsuka $\mathrm{M}$, et al. Elevated levels of oxidized low density 
lipoprotein show a positive relationship with the severity of acute coronary syndromes. Circulation 2001; 2001: 1955.

5. Ceriello A, Motz E. Is oxidative stress the pathogenic mechanism underlying insulin resistance, diabetes, and cardiovascular disease? The common soil hypothesis revisited. Arterioscler Thromb Vasc Biol 2004; 24: 816-23.

6. Vessby B, Uusitupa M, Hermansen K, Riccardi G, Rivellese AA, Tapsell LC, et al. Substituting dietary saturated for monounsaturated fat impairs insulin sensitivity in healthy men and women: the KANWU study. Diabetologia 2001; 44: 312-9.

7. Vessby B, Gustafsson I-B, Tengblad S, Boberg M, Andersson A. Desaturation and elongation of fatty acids and insulin action. Ann N Y Acad Sci 2002; 967: 183-95.

8. Rosell M, de Faire U, Hellénius M-L. Low prevalence of the metabolic syndrome in wine drinkers - is it the alcohol beverage or the lifestyle? Eur J Clin Nutr 2003; 57: $227-34$.

9. Sjogren P, Rosell M, Skoglund-Andersson C, Zdravkovic S, Vessby B, de Faire U, et al. Milk-derived fatty acids are associated with a more favourable LDL particle size distribution in healthy men. J Nutr 2004; 134: 1729-35.

10. Skoglund-Andersson C, Tang R, Bond MG, de Faire U, Hamsten A, Karpe F. LDL particle size distribution is associated with carotid intima-media thickness in healthy 50-year-old men. Arterioscler Thromb Vasc Biol 1999; 19: 2422-30.

11. Tholstrup T. Dairy products and cardiovascular disease. Curr Opin Lipidol 2006; 17: 1-10.

12. Azadbakht L, Mirmiran P, Esmaillzadeh A, Azizi F. Dairy consumption is inversely associated with the prevalence of the metabolic syndrome in Tehranian adults. Am J Clin Nutr 2005; 82: 523-30.

13. Liu S, Song Y, Ford ES, Manson JE, Buring JE, Ridker PM. Dietary calcium, vitamin D, and the prevalence of metabolic syndrome in middle-aged and older US women. Diabetes Care 2005; 28: 2926-32.

14. Liu S, Choi HK, Ford E, Song Y, Klevak A, Buring JE, et al. A prospective study of dairy intake and the risk of type 2 diabetes in women. Diabetes Care 2006; 29: 1279-84.

15. Rivellese AA, Maffettone A, Vessby B, Uusitupa M, Hermansen K, Berglund G, et al. Effects of dietary saturated, monounsaturated and n-3 fatty acids on fasting lipoproteins, LDL size and post-prandial lipid metabolism in healthy subjects. Atherosclerosis 2003; 167: 149-58.

16. Hodson L, Skeaff CM, Chisholm W-AH. The effect of replacing dietary saturated fat with polyunsaturated or monounsaturated fat on plasma lipids in free-living young adults. Eur J Clin Nutr 2001; 55: 908-15.

17. Lindström J, Louheranta A, Mannelin M, Rastas M, Salminen V, Erikssen J, et al. The Finnish Diabetes Prevention Study (DPS): lifestyle intervention and 3year results on diet and physical activity. Diabetes Care 2003; 26: 3230-6.
18. Sjogren P, Basu S, Rosell M, Silveira A, de Faire U, Vessby B, et al. Measures of oxidized low-density lipoprotein and oxidative stress are not related and not elevated in otherwise healthy men with the metabolic syndrome. Arterioscler Thromb Vasc Biol 2005; 25: 2580-6.

19. Basu S. Isoprostanes: novel bioactive products of lipid peroxidation. Free Rad Res 2004; 38: 105-22.

20. Sigurdardottir V, Fagerberg B, Hulthe J. Circulating oxidized low-density lipoprotein (LDL) is associated with risk factors of the metabolic syndrome and LDL size in clinically healthy 58 -year-old men (AIR study). J Intern Med 2002; 252: 440-7.

21. Hansel B, Giral P, Nobecourt E, Chantepie S, Bruckert E, Chapman MJ, et al. Metabolic syndrome is associated with elevated oxidative stress and dysfunctional dense high-density lipoprotein particles displaying impaired antioxidative activity. J Clin Endocrinol Metab 2004; 89: 4963-71.

22. Nälsén C, Vessby B, Berglund L, Uusitupa M, Hermansen K, Riccardi G, et al. Dietary (n-3) fatty acids reduce plasma F2-isoprostanes but not prostaglandin F2a in healthy humans. J Nutr 2006; 136: 1222-8.

23. Basu S, Helmersson J. Factors regulating isoprostane formation in vivo. Antioxid Redox Signal 2005; 7: $221-$ 35.

24. Lopez-Garcia E, Schulze MB, Manson JE, Meigs JB, Albert CM, Rifai N, et al. Consumption of (n-3) fatty acids is related to plasma biomarkers of inflammation and endothelial activation in women. J Nutr 2004; 134: 1806-11.

25. Ntambi JM, Miyazaki M. Recent insights into stearoylCoA desaturase-1. Curr Opin Lipidol 2003; 14: 255-61.

26. Ntambi JM, Miyazaki M, Stoehr JP, Kendziorski CM, Yandell BS, Song Y, et al. Loss of stearoyl-CoA desaturase-1 function protects mice against adiposity. Proc Natl Acad Sci U S A 2002; 99: 11482-6.

27. Dobrzyn P, Dobrzyn A, Miyazaki M, Cohen P, Asilmaz E, Hardie DG, et al. Stearoyl-CoA desaturase 1 deficiency increases fatty acid oxidation by activating AMP-activated protein kinase in liver. Proc Natl Acad Sci U S A 2004; 101: 6409-14.

28. Cohen P, Miyazaki M, Socci ND, Hagge-Greenberg A, Liedtke W, Soukas AA, et al. Role for stearoyl-CoA desaturase-1 in leptin-mediated weight loss. Science 2002; 297: 240-3.

29. Biddinger SB, Miyazaki M, Boucher J, Ntambi JM, Kahn CR. Leptin suppresses stearoyl-CoA desaturase 1 by mechanisms independent of insulin and sterol regulatory element-binding protein-1c. Diabetes 2006; 55: $2032-41$.

30. Jiang GQ, Li ZH, Liu F, Ellsworth K, Dallas-Yang Q, $\mathrm{Wu}$ M, et al. Prevention of obesity in mice by antisense oligonucleotide inhibitors of stearoyl-CoA desaturase-1. J Clin Invest 2005; 115: 1030-8.

31. Gutiérrez-Juárez R, Pocai A, Mulas C, Ono H, Bhanot $\mathrm{S}$, Monia BP, et al. Critical role of stearoyl-CoA desaturase-1 (SCD1) in the onset of diet-induced hepatic insulin resistance. J Clin Invest 2006; 116: 1686-95. 
32. Stumvoll M. Control of glycaemia: from molecules to men. Minkowski Lecture 2003. Diabetologia 2004; 47: $770-81$.

33. Attie AD, Krauss RM, Gray-Keller MP, Brownlie A, Miyazaki M, Kastelein JJ, et al. Relationship between stearoyl-CoA desaturase activity and plasma triglycerides in human and mouse hypertriglyceridemia. J Lipid Res 2002; 43: 1899-907.

34. Warensjö E, Öhrvall M, Vessby B. Fatty acid composition and estimated desaturase activities are associated with obesity and lifestyle variables in men and women. Nutr Metab Cardiovasc Dis 2006; 16: 128-36.

35. Warensjö E, Risérus U, Vessby B. Fatty acid composition of serum lipids predicts the development of the metabolic syndrome in men. Diabetologia 2005; 48: 1999-2005.

36. Riserus U, Tan GD, Fielding BA, Neville MJ, Currie J, Savage DB, et al. Rosiglitazone increases indexes of stearoyl-CoA desaturase activity in humans - link to insulin sensitization and the role of dominant-negative mutation in peroxisome proliferator-activated receptorgamma. Diabetes 2005; 54: 1379-84.

37. Yki-Järvinen H. Thiazolidinediones. N Engl J Med 2004; 351: 1106-18.

38. Kusunoki J, Kanatani A, Moller DE. Modulation of fatty acid metabolism as a potential approach to the treatment of obesity and the metabolic syndrome. Endocrine 2006; 29: 91-100.

Dr Rachel Fisher,

Atherosclerosis Research Unit,

King Gustaf V Research Institute,

Karolinska Institutet,

Karolinska University Hospital,

SE-I7I 76, Stockholm,

Sweden,

Tel: +46851773245 ,

Fax: +46831 1298 ,

E-mail: rachel.fisher@ki.se 